\title{
Beyond the Anatomy of Renal Nerves: Functional Diversity of Renal Nerves
}

\author{
Vishal N. Rao ${ }^{1,2} \cdot$ Marat Fudim $^{1,2} \cdot$ Jie Wang $^{3,4}$ \\ Published online: 25 February 2022 \\ (c) The Author(s), under exclusive licence to Springer Science+Business Media, LLC, part of Springer Nature 2022
}

Renal denervation (RDN) has been considered a very promising therapeutic option for patients with resistant hypertension (HTN) and uncontrolled hypertension. Early clinical studies demonstrated remarkable reductions in blood pressure among patients with elevated baseline systolic BP despite taking three or more antihypertensive medications [1, 2]. However, the first large sham-controlled clinical trial evaluating RDN did not demonstrate reduction in office systolic BP at 6 months [3], and another study failed to demonstrate consistent reductions in 24-h ambulatory BP at 3 months [4]. The most recent sham-controlled trial revealed significant heterogeneity of $\mathrm{BP}$ reduction at 3 months with unselected RDN in the absence of antihypertensive medications (SPYRAL HTN OFF-MED trial) [5]. Yet, unselected RDN with utilization of antihypertensive medications yielded varying results at 3-month and 6-month follow-up (SPYRAL HTN ON-MED) [6]. Both studies further confirmed the efficacy and safety of RDN but the amplitude of office systolic BP reduction was moderate: around $10 \mathrm{mmHg}$ because $20-30 \%$ patients were no-responders whose BP was not decreased or even increased after RDN. This may counteract BP-lowering effects achieved by RDN. Comparisons of different RDN methods have also yielded varying results. Among patients with resistant hypertension, endovascular ultrasound-based RDN demonstrated superiority to radiofrequency ablation of the main renal arteries only, whereas a combined approach of radiofrequency ablation of the main arteries, accessories, and side branches proved no difference

Jie Wang

jay329329@yahoo.com

1 Department of Cardiology, Duke University Medical Center, Durham, NC 27710, USA

2 Duke Clinical Research Institute, Durham, NC 27710, USA

3 Department of Cardiology, College of Physicians \& Surgeons, Columbia University, New York, NY 10032, USA

4 Jiangsu Province Hospital, Nanjing 210029, Jiangsu Province, China
(RADIOSOUND-HTN) [7]. It has been demonstrated that an approximately $30 \%$ non-responder rate was consistently observed among various energies-based RDN [8]. These cumulative findings have motivated the scientific community and medical device industry to re-evaluate the subtle implications of renal vascular anatomy, physiological responses to renal nerve stimulations, and differences in device and trial design. This recognition that RDN can produce heterogeneous afferent sympathetic effects has both changed therapeutic goals and revitalized the potential of therapeutic RDN to provide significant clinical benefits by closely examining the renal neurovascular interactions.

Reevaluating anatomical neurovascular relationships of the renal artery has opened new pathways towards better understanding RDN as potential therapies. Garcia-Touchard et al. performed quantitative analysis of the human renal nervous system anatomy which defined key patterns [9]. Contrary to prior understandings, only $17 \%$ of cases had renal innervation closely following the main renal artery, with the majority of renal nerves ( $>50 \%)$ bypassing the main renal artery when reaching the kidney. Perivascular space also consisted of plexuses, fused ganglia, or branches from sympathetic chains [9]. This observed heterogeneity in anatomic structures offers clues that inform physiologic relationships between the nervous system and renal artery.

In this context, Zhao et al. characterized blood pressure patterns by mapping renal innervations through renal nerve stimulation (RNS) [10]. In summary, the authors categorized phenotypic responses of blood pressure and heart rate changes to RNS in 24 Chinese Kunming dogs across a total of 483 stimulated sites [10]. The authors identified five unique and important blood pressure responses: (1) a continuous ascending and stabilized blood pressure response above baseline (27\%); (2) a declining followed by rise over baseline blood pressure response (11.8\%); (3) a decline, followed by rise below baseline response $(14 \%)$; (4) a fluctuating blood pressure response close to baseline (40\%); and (5) 
Fig. 1 Theoretical framework for the functional nature of renal nerves: red lines/dots represent "hot spots"- pressor spots. These are nerves that raise the blood pressure when stimulated. They are the ideal target of renal denervation. Green line/spots represent "cold spots"-inhibitory spots, which lower the blood pressure when stimulated. The nerve fibers in yellow are neutral in their contribution for blood pressure physiology and do not show hemodynamic effects when stimulated. From Fudim et al. [11]

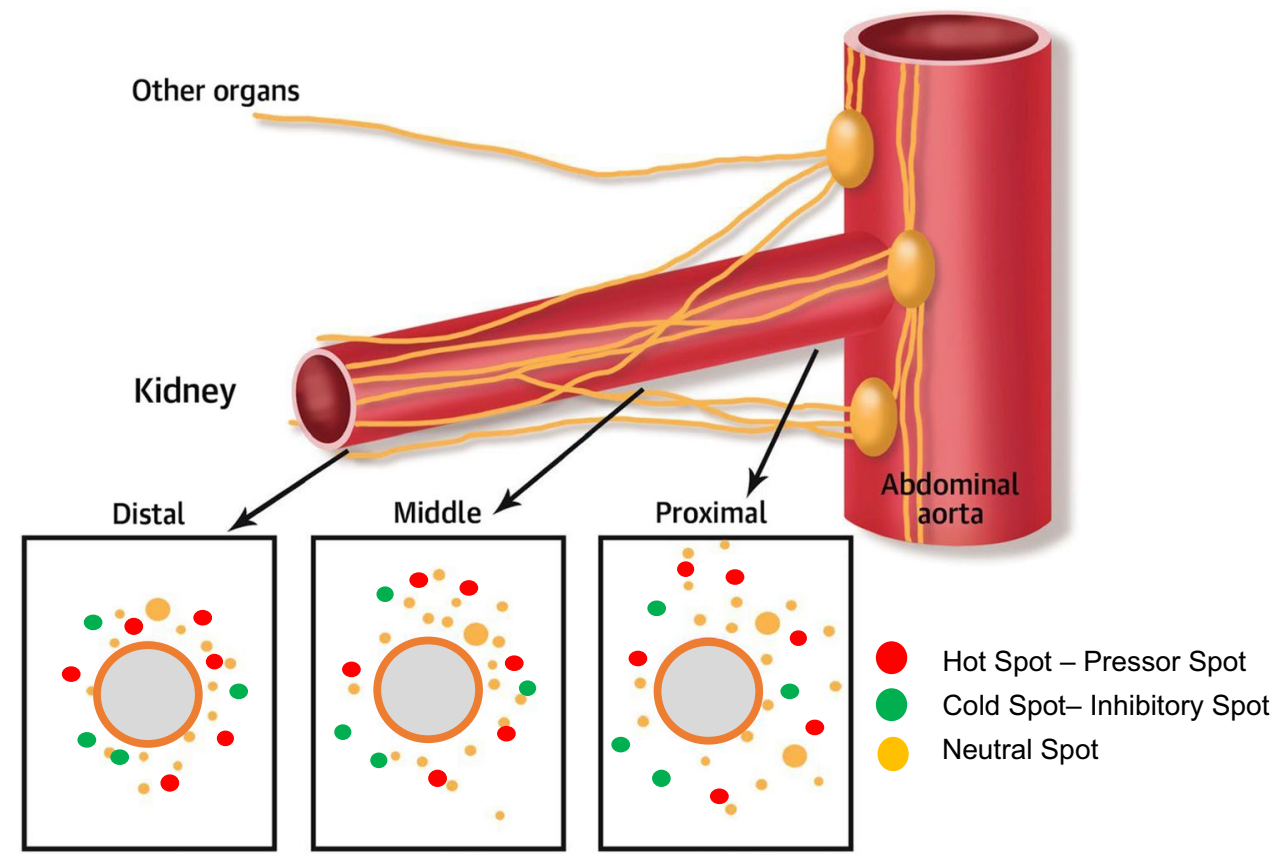

a continuous decline and stabilized blood pressure response below baseline (7.2\%).

These findings offer important clues to the possible physiologic effects of anatomical variability of renal innervation, potentially distinguishing excitatory versus inhibitory nerve clusters that may have previously limited sustained efficacy of nonspecific RDN (Fig. 1). As the authors importantly pointed out, these data do not confirm the clear relationships between physiologic blood pressure responses and anatomic patterns of renal artery innervation as described on histologic analyses [10]. Yet, targeting certain types of blood pressure responses may produce consistent and expected patterns in clinical RDN. The potential applications of these findings in clinical practice are warranted [11].

\section{References}

1. Krum, H., Schlaich, M., Whitbourn, R., et al. (2009). Catheterbased renal sympathetic denervation for resistant hypertension: A multicentre safety and proof-of-principle cohort study. Lancet, $373,1275-1281$.

2. Esler, M. D., Krum, H., Sobotka, P. A., et al. (2010). Renal sympathetic denervation in patients with treatment-resistant hypertension (The Symplicity HTN-2 Trial): A randomised controlled trial. Lancet, 376, 1903-1909.

3. Bhatt, D. L., Kandzari, D. E., O'Neill, W. W., et al. (2014). A controlled trial of renal denervation for resistant hypertension. $N$ Engl J Med, 370, 1393-1401.
4. Mathiassen, O. N., Vase, H., Bech, J. N., et al. (2016). Renal denervation in treatment-resistant essential hypertension. A randomized, SHAM-controlled, double-blinded 24-h blood pressurebased trial. J Hypertens, 34, 1639-47.

5. Townsend, R. R., Mahfoud, F., Kandzari, D. E., et al. (2017). Catheter-based renal denervation in patients with uncontrolled hypertension in the absence of antihypertensive medications (SPYRAL HTN-OFF MED): A randomised, sham-controlled, proof-of-concept trial. Lancet, 390, 2160-2170.

6. Kandzari, D. E., Bohm, M., Mahfoud, F., et al. (2018). Effect of renal denervation on blood pressure in the presence of antihypertensive drugs: 6-month efficacy and safety results from the SPYRAL HTN-ON MED proof-of-concept randomised trial. Lancet, 391, 2346-2355.

7. Fengler, K., Rommel, K. P., Blazek, S., et al. (2019). A three-arm randomized trial of different renal denervation devices and techniques in patients with resistant hypertension (RADIOSOUNDHTN). Circulation, 139, 590-600.

8. Townsend, R. R., \& Sobotka, P. A. (2018). Catheter-based renal denervation for hypertension. Curr Hyp Rep, 20, 93.

9. García-Touchard, A., Maranillo, E., Mompeo, B., \& Sañudo, J. R. (2020). Microdissection of the human renal nervous system: Implications for performing renal denervation procedures. Hypertension, 76, 1240-1246.

10 Zhou, H., Li, Y., Xu, Y., et al. (2021). Mapping renal innervations by renal nerve stimulation and characterizations ofblood pressure response patterns. J Card Trans Res.

11 Fudim, M., Sobotka, A. A., Yin, Y. H., et al. (2018). Selective vs. global renal denervation: A case for less is more. Curr Hyp Rep, 20,37 .

Publisher's Note Springer Nature remains neutral with regard to jurisdictional claims in published maps and institutional affiliations. 